\title{
ENFERMAGEM EM PRÁTICAS AVANÇADAS NO ATENDIMENTO PRÉ-HOSPITALAR: OPORTUNIDADE DE AMPLIAÇÃO DO ACESSO NO BRASIL
}

Marisa Aparecida Amaro Malvestio ${ }^{1}$ Lilian Prates Belem Behringer ${ }^{1}$ Sérgio Dias Martuchi ${ }^{1}$

Marcos Aurélio da Silva Fonseca ${ }^{1}$ Luciano Silva ${ }^{1}$

Eduardo Fernando de Souza ${ }^{1}$ Gilson Clementino Hanszman ${ }^{1}$ Rildo Bezerra ${ }^{1}$

\author{
https://orcid.org/0000-0003-0633-9278 \\ https://orcid.org/0000-0003-004492 \\ https://orcid.org/0000-0002-6873-4647 \\ https://orcid.org/0000-0002-3031-4700 \\ https://orcid.org/0000-0003-1748-028 \\ https://orcid.org/0000-0002-2108-6507 \\ https://orcid.org/0000-0002-7934-3406 \\ https://orcid.org/0000-0002-4806-7824
}

Objetivo: analisar o cenário de implementação da Enfermagem de Praticas Avançadas (EPA) no atendimento pré-hospitalar (APH) como ferramenta de acesso ao cuidado no Brasil. Metodologia: Estudo exploratório, analítico, de revisão narrativa, delineado para constituir uma reflexão teórica sobre o cenário de aplicação da EPA no modelo pré-hospitalar brasileiro a partir das experiências de outros países e da análise do modelo e resultados da atenção pré-hospitalar no Brasil. Conclusão: Experiências internacionais demonstram que a EPA é uma estratégia de valor na busca de acesso à saúde A ampliação do papel dos enfermeiros no modelo brasileiro de APH pode viabilizar o alcance da cobertura $100 \%$ e incrementar a capacidade de avaliação e a realização de procedimentos em tempo adequado, assegurando acesso oportuno ao cuidado.

Descritores: Serviços Médicos de Emergência; Prática Avançada de Enfermagem; Assistência à Saúde

\section{NURSING IN ADVANCED PRACTICES IN PRE-HOSPITAL CARE: OPPORTUNITY TO EXPAND ACCESS TO CARE IN BRAZIL}

Objective: to analyze the scenario of implementation of Advanced Practice Nursing (APN) in prehospital care (PHC) as a tool for access to care in Brazil. Methodology: Exploratory, analytical, narrative review study, designed to constitute a theoretical reflection on the scenario of application of APN in the Brazilian pre-hospital model from the experiences of other countries and the analysis of the model and results of pre-hospital care in Brazil. Conclusion: International experiences demonstrate that the APN is a valuable strategy in the search for access to health The expansion of the role of nurses in the Brazilian model of PHC can enable the reach of $100 \%$ coverage and increase the capacity for evaluation and the performance of procedures in adequate time, ensuring timely access to care.

Descriptors: Emergency Medical Services; Advanced Nursing Practice; Health Care

\section{ENFERMERİA EN PRÁCTICAS AVANZADAS EN ATENCIÓN PREHOSPITALARIA: OPORTUNIDAD DE AMPLIAR EL ACCESO A LA ATENCIÓN EN BRASIL}

Objetivo: analizar el escenario de implementación de la Enfermería de Práctica Avanzada (EPA) en la atención prehospitalaria (APH) como herramienta de acceso a la atención en Brasil. Metodología: estudio exploratorio, analítico, de revisión narrativa, diseñado para constituir una reflexión teórica sobre el escenario de aplicación de EPA en el modelo prehospitalario brasileño a partir de las experiencias de otros países y el análisis del modelo y los resultados de la atención prehospitalaria en Brasil Brasil Conclusión: Las experiencias internacionales demuestran que la EPA es una estrategia valiosa en la búsqueda de acceso a la salud. La expansión del papel de las enfermeras en el modelo brasileño de APS puede permitir alcanzar el 100\% de cobertura y aumentar la capacidad de evaluación y el desempeño de los procedimientos en tiempo adecuado, asegurando el acceso oportuno a la atención.

Descriptores: Servicios médicos de emergencia; Práctica de enfermería avanzada; Cuidado de la salud

${ }^{1}$ Comissão Nacional de Urgência e Emergência do Conselho Federal de Enfermagem.

Autor correspondente: Marisa Amaro Malvestio - marisa.malvestio@gmail.com 


\section{INTRODUÇÃO}

O desenvolvimento da Enfermagem em Práticas Avançadas (EPA) é uma das estratégias globais de estímulo à ampliação do acesso e da cobertura em saúde. A proposta é divulgada pela Organização Mundial de Saúde (OMS) e apoiada pela Organização Pan-americana de Saúde (OPAS) e pelo Conselho Federal de Enfermagem (COFEN) ${ }^{(1-5)}$.

A EPA ${ }^{(1)}$ é definida como "enfermeiros que adquiriram conhecimento especializado, habilidades para tomada de decisão em situações complexas e competências clínicas para prática expandida, cujas características são moldadas pelo contexto ou país em que estão credenciados para praticar". Trata-se de um conceito que evoca a ampliação do escopo de práticas para domínios de alta especificidade, compatibilizados às necessidades de saúde da sociedade e seus cidadãos, respeitando políticas públicas e o desenvolvimento da profissão em cada país ${ }^{(1-2: 5)}$.

A EPA na Atenção Primária à Saúde (APS) é uma potente estratégia para o alcance de cobertura e acesso universal ${ }^{(6-7)}$. No entanto, estudos têm demonstrado que as possibilidades de aplicação do conceito são amplas e a área de urgência é uma das futuras direções ${ }^{(1)}$.

O cenário da atenção às urgências no Brasil é marcado por desafios típicos de países em desenvolvimento com múltiplas transições em andamento(8:11). Apesar da implementação da Rede de Atenção às Urgências (RUE)(9), há necessidade de viabilizar cobertura $100 \%$ e atendimento oportuno aos agravos tempo-dependentes, bem como garantir cuidado nos quadros de agudização de causas crônicas advindas do envelhecimento da população que ainda convive com as doenças infecciosas e parasitárias ${ }^{(8: 10)}$.

$\mathrm{O}$ atendimento pré-hospitalar (APH) é um dos pontos assistenciais da RUE e a mais democrática das portas de entrada do Sistema Único de Saúde (SUS), pois cidadãos de todas as classes sociais, cobertos ou não por planos privados, podem necessitar de cuidado de urgência. Nessa fase do atendimento, o "tempo até o cuidado adequado" influencia o resultado de saúde e assim, "cobertura e acesso" ganham novas dimensões e interagem de maneira pragmática. A "cobertura " de um serviço de APH não envolve apenas a disponibilidade territorial do serviço, mas associa "disponibilidade em tempo adequado". Já o "acesso oportuno", associa "cuidado adequado em tempo adequado". Para um ótimo APH, é preciso explorar soluções que garantam acesso oportuno ao cidadão e não apenas cobertura.

Objetivo analisar o cenário de implementação da EPA no âmbito do APH como ferramenta de ampliação da cobertura e acesso oportuno ao cuidado de urgência e emergência no modelo brasileiro.

\section{METODOLOGIA}

Trata-se de um estudo exploratório, analítico, de revisão narrativa, delineado para constituir uma reflexão teórica sobre o cenário da aplicação da EPA na ampliação de acesso ao cuidado pré-hospitalar no Brasil. A revisão se inicia com a análise das experiências de implantação da EPA em outros países e especificamente nas urgências, para em seguida, analisar aspectos do modelo implementado e do cenário e resultados da atenção pré-hospitalar no Brasil.

\section{Enfermagem em Práticas Avançadas pelo mundo}

A OMS está estimulando a revisão de papéis entre os profissionais de saúde, para alcançar uma combinação melhor de competências e atribuições e adequar a oferta de cuidado, ampliando o acesso. Nessa proposta, há espaço para uma nova conformação da prática do Enfermeiro e uma expansão em seu papel. ${ }^{(1-6)}$

Experiências com EPA existem desde 1940, principalmente para atender as necessidades de saúde em áreas rurais e remotas, onde a disponibilidade de médicos era crítica. A partir de 1990, inúmeros países redefiniram a atuação do enfermeiro em áreas onde precisavam ampliar a resolubilidade, como a APS, cuidado domiciliar e crítico, obstetrícia, saúde do idoso e da criança. ${ }^{(1-6)}$

Começam a surgir bons resultados de pesquisa sobre o impacto da EPA e revisões sistemáticas têm mostrado que enfermeiros promovem cuidados seguros e eficazes, melhoram o acesso a serviços e reduzem tempos de espera. com ótima qualidade de atendimento e maior eficiência do sistema de saúde. (1:3-4:11) $^{-11}$

Delamaire e Lafortune (2010)(1) analisaram os resultados da atuação de EPA na APS em 12 países. A avaliação foi focada no impacto sobre o cuidado ao paciente (acesso, qualidade, resultados e satisfação) e sobre o custo. Mesmo em diferentes cenários, os resultados mostraram melhor acesso à serviços e redução de tempos de espera, com ótima qualidade de atendimento para diferentes agravos. Pacientes expressaram alta taxa de satisfação, principalmente pela disponibilidade dos enfermeiros para fornecer informações e aconselhamento. Os resultados foram neutros quanto aos custos ou com redução discreta em comparação com abordagens de substituição de tarefas com médicos. No entanto, a redução de custos potenciais relacionadas à prevenção de complicações e o sobre a integração de serviços não foi considerada.

A revisão sistemática de Newhouse et al (2011)(11) avaliou se desfechos de cuidados por enfermeiros eram semelhantes aos desfechos por outros provedores e demonstrou que os cuidados prestados pelos enfermeiros em colabo- 
ração com os médicos, são semelhantes e, de certa forma, melhores do que aqueles prestados apenas pelos médicos. Nos agravos agudos, a abordagem do enfermeiro foi associada com redução do tempo de internação e custo para pacientes hospitalizados. Os autores concluíram que os cuidados realizados por enfermeiros foram eficazes e de alta qualidade.

Pesquisadores canadenses(3) analisaram o custo-efetividade das ações de enfermeiros treinados, com bons resultados em: cuidado hospitalar, ambulatorial e de transição; qualidade da assistência e redução de custos (a partir da redução de tempo de internação e de reinternações de idosos); menos visitas à emergência por pacientes oncológicos e bom impacto na satisfação do paciente. A redução de erros e a redução de cuidados abaixo do padrão ideal também foram associados à participação dos enfermeiros no cuidado.

\section{Enfermagem em Práticas Avançadas nas urgências}

A EPA nas urgências hospitalares já está bem estabelecida em países como Irlanda, Austrália, Canadá, Polônia, Inglaterra e Bélgica(1). Nesse espectro, o APH também pode ser considerado uma área de expansão da atuação do enfermeiro por sua complexidade e especificidade. Experiências com enfermeiros no APH já são encontradas em alguns países $^{(12,13,14)}$ onde, não obstante a continuidade do trabalho de outros profissionais, já são encontrados resultados efetivos em termos de cuidado e estratégia de acesso.

Em Portugal ${ }^{(12)}$, o APH possui duas modalidades com enfermeiros: a ambulância de Suporte Imediato de Vida, (também tripulada por um técnico de ambulância), e as Viaturas Médicas de Emergência e Reanimação, (veículos tripulados também por médicos). O Suporte Imediato foi idealizado para o atendimento, estabilização e transporte de vítimas de acidentes e doença súbita e já atende $97 \%$ dos pacientes críticos do país ${ }^{(12)}$.

$\mathrm{Na}$ Suécia ${ }^{(13)}$, em 2000, foi determinado que todas as ambulâncias deveriam ser tripuladas por um Enfermeiro, fato alcançado progressivamente em 2005. O objetivo foi aumentar as competências em saúde no APH. Em 2015, após 10 anos dessa medida, pesquisadores demonstraram que houve melhora na qualidade do cuidado frente ao aperfeiçoamento da avaliação clínica e do exame físico que viabilizaram a tomada de decisões e realização de procedimentos por enfermeiros na cena.Tais melhorias levaram a um cuidado resolutivo e baseado nas necessidades do paciente ${ }^{(13)}$.

$\mathrm{Na}$ Holanda(14), ambulâncias atuam com enfermeiros e apoio médico quando necessário. Desde 1992, os profissionais recebem treinamento e atuam com protocolos con- sensuados entre associações médicas e de enfermagem. Além do curso inicial, os profissionais recebem treinamentos regionais e nacionais com exames teóricos anuais. Nesse modelo, estudo(14) analisou as competências médicas e de enfermagem disponíveis no APH e considerou que o enfermeiro possui ampla gama de competências sobrepostas às dos médicos e nessa perspectiva, as competências adicionais do médico, puderam ser adicionadas complementarmente.

Nos Estados Unidos ${ }^{(15)}$, o Corpo de Bombeiros de Los Angeles detectou um aumento nas solicitações de baixa complexidade com alta reincidência. Fatores como o envelhecimento da população, sobrecarga de pacientes sem acesso ao sistema de saúde, excessivo acionamento para agravos em saúde mental e casos sociais eram a causa. Para esse cenário, além do atendimento às urgências, o serviço adotou uma abordagem proativa e desenvolveu um sistema para fornecer atendimento e encaminhamento adequados, com unidades especializadas, tripuladas por bombeiro ou paramédico e um enfermeiro, com abordagem resolutiva e encaminhamento a unidades primárias. Os resultados demonstraram eficácia nos quadros agudos em idosos, usuários frequentes e pacientes com problemas mentais, resultando em redução da sobrecarga dos recursos de emergência ${ }^{(15)}$.

\section{O contexto das urgências e da atenção pré-hospitalar no Brasil \\ O modelo brasileiro de atenção às urgências perma-} nece fragmentado, subfinanciado e reativo às condições agudas ou à agudização das crônicas. Custos elevados afligem os municípios e é preocupante a baixa disponibilidade e/ou a baixa adesão de médicos nos serviços de urgência. A transição demográfica eleva o percentual de idosos na população e traz os quadros repetitivos de agudização das condições crônicas para o convívio com as doenças tempo-dependentes e causas externas e infecciosas que já superlotam as portas de urgência. A crise econômica e a redução de empregos formais movimentam milhões de usuários do sistema privado para o SUS, cuja porta de entrada, historicamente mais fácil e transponível, é da urgência. Como resultado de todos esses fatores temos portas de urgência superlotadas ${ }^{(9-10)}$

Para o enfrentamento a esse cenário, vem sendo implementada a RUE ${ }^{(9-10)}$, que preconiza a cooperação e a formação de arranjos organizativos entre os diferentes serviços no mesmo território, para ampliar a disponibilidade de recursos, com economia de escala. Na RUE, o atendimento à urgência é responsabilidade de todos os pontos de atenção, desde o 
APH móvel, e a APS até as portas de urgência e a atenção domiciliar, respeitada a capacidade de atenção de cada um ${ }^{(9-10)}$.

Em 2003, foi estabelecido um modelo de sistema de APH móvel para o país, com dígito telefônico único, regulação médica, veículos padronizados, equipamentos e modalidades. No ambiente público, com essa estrutura foi criado o Serviço de Atendimento Móvel de Urgência ${ }^{(16-17)}$, o SAMU 192, cujo objetivo é chegar precocemente à vítima de agravo de urgência ou emergência de qualquer natureza, realizando o atendimento e o transporte às unidades que prestam serviços de urgência. $O$ fomento federal para adesão ao modelo, com financiamento e custeio tripartite, estimulou sua rápida implementação em todo o país ${ }^{(16-17)}$.

Além do papel assistencial, o SAMU 192 é fundamental na performance da RUE, funcionando como ligação entre os pontos assistenciais e organizador do fluxo de atenção a partir do exercício da regulação das urgências, tornando-se uma poderosa ferramenta de acesso ao cuidado para o cidadão em situação de urgência. Assim, a configuração do SAMU 192 tem potencial para afetar seus próprios resultados assistenciais e, também, os resultados da RUE(9-10).

As modalidades assistenciais do SAMU 192 são: o SUPORTE BÁSICO DE VIDA (SBV), cuja equipe mínima é composta por dois profissionais (um técnico ou auxiliar de enfermagem e um condutor), e o SUPORTE AVANÇADO DE VIDA (SAV) com três profissionais (um médico, um enfermeiro e um condutor) ${ }^{(16-17)}$

Em 2018(18), o SAMU 192 possuía 192 centrais de regulação, estava presente em 3.533 municípios (63,4\% do total), (Figura 1) e alcançava cobertura de 170,6 milhões de habitantes $(82,2 \%)$.

Figura 1: Cartograma representando a cobertura do SAMU 192 segundo os limites das cidades que aderiram ao sistema. Brasil, junho/2018.

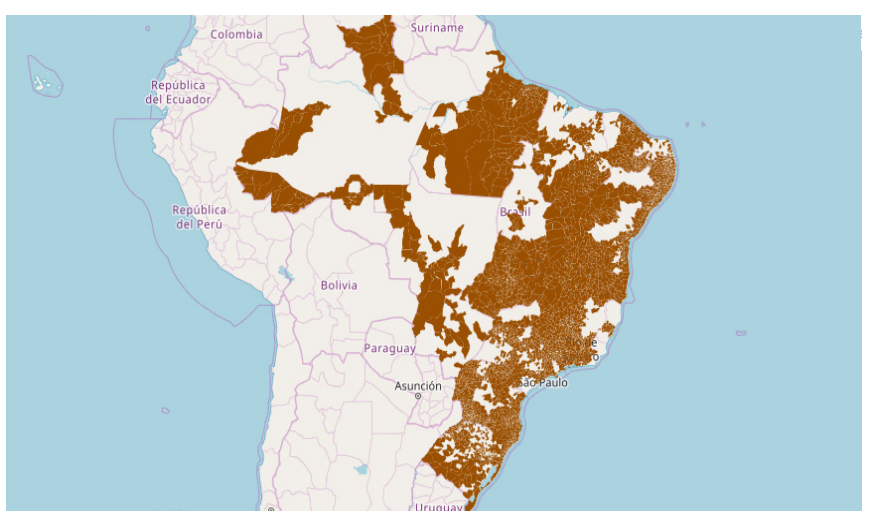

Fonte: INDE19
A ampla cobertura do SAMU 192 se justifica pela forte presença nas capitais e centros urbanos. Estados como Santa Catarina, Goiás, Roraima, Paraíba, Alagoas, Sergipe e o Distrito Federal alcançaram 100\% de cobertura em seu território, porém para alcançar $100 \%$ de cobertura no país restam 2037 municipios (36,6\%) e cerca de 38 milhões de habitantes (17,8\%) dispersos em áreas rurais extensas e em municipios com menos de 50 mil habitantes principalmente no centro-oeste, norte e nordeste ${ }^{(19-20)}$.

A Figura 2 apresenta a evolução do SAMU 192 nos últimos 6 anos. Em 2018, o modelo contava com 3.307 ambulâncias, sendo 2.702 de SBV (81,7\%) e 605 de SAV (18,3\%) ${ }^{(19-20)}$. A resposta aérea, por embarcação e por motos não será avaliada no presente estudo.

Figura 2: Evolução do número de municípios cobertos e da distribuição das unidades móveis de SBV e SAV do SAMU 192. Brasil, 2013-2018

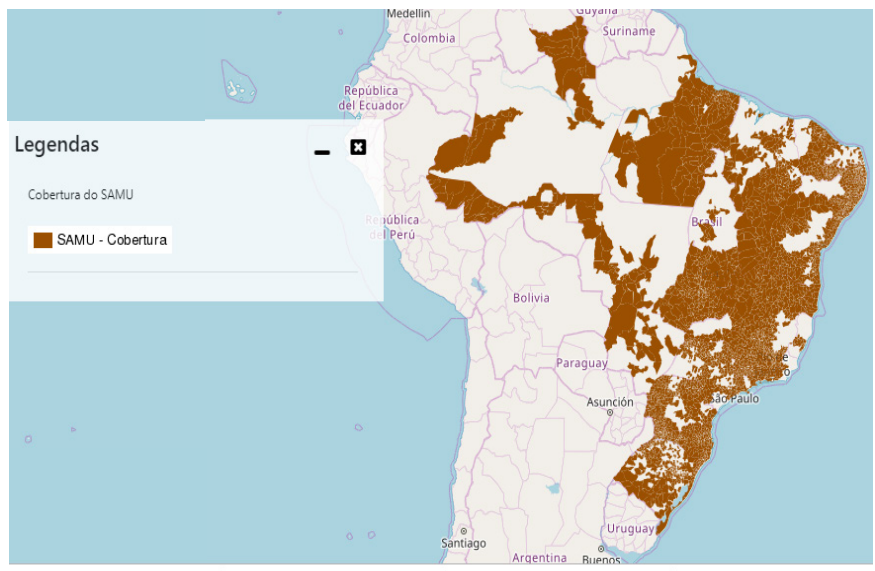

Fonte: SAGE (2013 a 2017) ${ }^{(20)}$ e Ministério da Saúde (2018) ${ }^{(18)}$

No periodo, 415 novas ambulâncias de SBV e 51 ambulâncias de SAV foram implementadas na cobertura de atenção pré-hospitalar de 769 cidades, gerando cobertura para cerca de 29,5 milhões de pessoas ${ }^{(20)}$. Para esse crescimento houve fomento à regionalização(21), para viabilizar economia de escala para custeio e manutenção do serviço.

Os indicadores utilizados na avaliação de cobertura geralmente são populacionais. No início da implantação do SAMU 192 a legislação recomendava um SBV para cada 100 mil habitantes e um SAV para cada 400 mil $^{(16: 21)}$. Na evolução do modelo, com a necessidade de ampliar cobertura e melhorar "tempo-resposta", foram associados critérios geográficos, de mobilidade, de disponibilidade de recursos em saúde e possibilidade de estabelecimento de economia de escala(16-17,21) 
Nesse cenário, a Figura 3 demonstra que proporção das modalidades assistenciais e a proporção das cidades cobertas vem se modificando.

Figura 3: Evolução da proporção de unidades móveis segundo a modalidade e municípios cobertos do SAMU 192. Brasil, 2013-2018.

\begin{tabular}{llll} 
& 2003 & 2013 & 2018 \\
\hline Unidade SAV:Unidade SBV & $1: 4$ & $1: 4,1$ & $1: 4,4$ \\
\hline Unidade SBV:Municipio & - & $1: 1,2$ & $1: 1,3$ \\
\hline Unidade SAV:Município & - & $1: 4,9$ & $1: 5,8$
\end{tabular}

Fonte: SAGE (2013 a 2017) ${ }^{(20)}$ e Ministério da Saúde (2018) ${ }^{(18)}$

Observam-se duas tendências: crescimento da cobertura e da capacidade de resposta na direção da modalidade SBV e a perspectiva de compartilhamento de uma unidade entre várias cidades frente a política de regionalização. No caso do SAV, cada unidade já atende a 5,8 municípios e esse cenário pode ser pior, se considerarmos que as capitais e grandes cidades possuem múltiplas unidades, agravando a proporção em áreas do interior.

Quando uma ambulância realiza a cobertura de múltiplas cidades, as distâncias a serem percorridas são maiores, seja no deslocamento até o paciente ou até a unidade de saúde de destino. Em grandes distâncias, o tempo-resposta ${ }^{(17)}$ (tempo entre o acionamento do sistema de urgência e a chegada da ambulância para atendimento) e o tempo total ${ }^{(17)}$ (tempo entre um acionamento e liberação para um novo atendimento) se tornam ainda maiores, ampliando o tempo de ocupação da viatura com deslocamentos e limitando a disponibilidade da ambulância como recurso assistencial. Esse panorama constitui cobertura com falso acesso ao cuidado, pois restringe e atrasa o cuidado em tempo adequado.

Esse crescimento na direção do SBV é preocupante frente a capacidade resolutiva da modalidade. O SBV é a primeira resposta às solicitações de socorro simplesmente por ser a mais disponivel, porém nele atuam 2 profissionais de nível médio(16-17). A despeito da importância do SBV, seus profissionais, mesmo treinados, vivenciam situações para além da capacidade de abordagem, pois suas competências e prerrogativas profissionais são restritas e essa é uma perspectiva temerosa para a segurança assistencial. Por sua vez, o SAV concentra a capacidade clínica e reso- lutiva, mas responde por $18,3 \%$ da força de trabalho e não está disponivel de forma oportuna e regular no território.

Contribuem para esse cenário de polarização entre o SAV e o SAV, o design do modelo e custos (ou dificuldades) com recursos humanos e manutenção do SAV (materiais, medicamentos e equipamentos). O desinteresse ou indisponibilidade de médicos para ocupar as vagas nos centros urbanos e/ou os custos de sua fixação em territórios rurais ou remotos, também faz com que os gestores prefiram investir na implantação de SBV(21).

A Figura 4 apresenta a distribuição dos atendimentos realizados pelo SAMU 192, em 2018, e factualmente apresenta a participação das modalidades na assistência.

Figura 4: Distribuição de atendimentos e transporte interunidades, segundo a modalidade de atendimento. SAMU 192, Brasil, Jan-Dez, 2018.

\begin{tabular}{|c|c|c|c|c|c|c|}
\hline & \multicolumn{2}{|c|}{$\begin{array}{l}\text { Atendimento pré- } \\
\text {-hospitalar terrestre }\end{array}$} & \multicolumn{2}{|c|}{$\begin{array}{l}\text { Transporte inter-u- } \\
\text { nidades de saúde }\end{array}$} & \multicolumn{2}{|c|}{$\begin{array}{l}\text { Total de atendi- } \\
\text { mentos terrestres }\end{array}$} \\
\hline & $N$ & $\%$ & $N$ & $\%$ & $\mathrm{~N}$ & $\%$ \\
\hline SAV & 767.009 & $17,4 \%$ & 253.775 & $34,2 \%$ & 1.020 .784 & $19,8 \%$ \\
\hline SBV & 3.639 .876 & $82,6 \%$ & 487.070 & $65,8 \%$ & 4.126 .946 & $80,2 \%$ \\
\hline TOTAL & 4.406 .885 & $100 \%$ & 740.845 & $100 \%$ & 5.147 .730 & $100 \%$ \\
\hline
\end{tabular}

Fonte: Tabnet DATASUS ${ }^{(22)}$, acessado em 15/04/19.

O SBV efetivou 82,6\% dos atendimentos e o transporte interunidades correspondeu a um a cada oito acionamentos dessa modalidade. Já o SAV ocupou 1/4 de sua disponibilidade em transportes, que geralmente envolve longas distâncias, maior tempo de deslocamento e períodos de espera, resultando em mais indisponibilidade para os atendimentos.

Apesar da belissima trajetória do SAMU 192 na história do SUS, com reconhecimento pela população e amplo percentual de cobertura informado, o acesso oportuno ao cuidado de urgência permanece fora do alcance para milhões de pessoas. As dificuldades são políticas, econômicas e derivadas do modelo escolhido. Há uma necessidade urgente de ampliar cobertura, viabilizando o serviço em cidades menores, mas há também a necessidade de garantir segurança assistencial, ampliando a capacidade resolutiva das equipes disponiveis. 
Espaço para a EPA como estratégia para acesso oportuno no APH

No modelo brasileiro de APH, a participação do enfermeiro está restrita à composição do SAV e a coordenação da equipe de enfermagem ${ }^{(16-17)}$. No entanto, considerando as competências e as prerrogativas profissionais que podem ser incorporadas, a introdução do enfermeiro qualificando o SBV como 30 componente, ajustaria a polarização existente entre as competências em saúde disponíveis no SBV e no SAV, compatibilizando a capacidade da equipe às potenciais necessidades dos pacientes $^{(5)}$. Nessa composição alcança-se melhor capacidade de avaliação com pouca incorporação tecnológica, viabilizando a realização segura de orientações, procedimentos e administração de medicamentos em situações restritas por regulação, telemedicina e protocolos, visando a estabilização de pacientes de maior complexidade e assim, potencial melhoria na atenção à agravos crônico-agudizados e tempo-dependentes ${ }^{(5)}$

Os enfermeiros já participam do APH brasileiro. Dados do sistema de registro de produção do Ministério da Saúde já registram a força e a direção dessa participação (Figura 5).

Figura 5: Distribuição dos atendimentos e transporte interunidades, segundo a modalidade de atendimento e profissionais envolvidos. SAMU 192, Brasil, Jan-Dez, 2018.

\begin{tabular}{|c|c|c|c|c|c|c|c|c|}
\hline & \multicolumn{4}{|l|}{ SAV } & \multicolumn{4}{|l|}{ SBV } \\
\hline & \multicolumn{2}{|c|}{ Atendimento } & \multicolumn{2}{|c|}{ Transporte } & \multicolumn{2}{|c|}{ Atendimento } & \multicolumn{2}{|c|}{ Transporte } \\
\hline & $\mathrm{N}$ & $\%$ & $\mathrm{~N}$ & $\%$ & $\mathrm{~N}$ & $\%$ & $\mathrm{~N}$ & $\%$ \\
\hline $\begin{array}{l}\text { Com } \\
\text { Médico }\end{array}$ & 560.960 & 73,1 & 177.110 & 69,8 & - & - & 15.797 & 3,2 \\
\hline $\begin{array}{l}\text { Com } \\
\text { Enfer- } \\
\text { meiro* }\end{array}$ & 206.049 & 26.9 & 76.665 & 30,2 & - & - & 48.774 & 10,0 \\
\hline $\begin{array}{l}\text { Com } \\
\text { Téc. } \\
\text { Enf. }\end{array}$ & - & - & - & - & 2.945 .291 & 80,9 & 350.063 & 71,9 \\
\hline $\begin{array}{l}\text { Com } \\
\text { Aux. } \\
\text { Enf }\end{array}$ & - & - & - & - & 694.585 & 19,1 & 72.436 & 14,9 \\
\hline Total & 767.009 & 100 & 253.775 & 100 & 3.639 .876 & 100 & 487.0770 & 100 \\
\hline
\end{tabular}

Fonte: Tabnet DATASUS(22) em 15/04/19. *Com enfermeiro, sem médico.

Em 2018, compondo o SAV com o condutor, o enfermeiro realizou $26,9 \%$ dos atendimentos e $30,2 \%$ dos transportes. No SBV compondo com o condutor, o enfermeiro efetivou $10 \%$ dos transportes. Esses dados representam a força da presença do enfermeiro no território, sua aderência ao APH e sua importância como agente de acesso ao cuidado. Por outro lado, esse registro demonstra que 1/4 dos atendimentos e $1 / 3$ dos transportes registrados como SAV, estão sendo realizados sem a presença de médicos. É possivel que a indisponibilidade de médicos no território, baixa adesão e alta rotatividade justifiquem esses resultados, assim como já visto em outros países ${ }^{(1-3)}$. Devido às regras de registro, não foi possivel analisar a frequência em que enfermeiro e técnico ou auxiliar atuam em conjunto.

Observa-se que o auxiliar de enfermagem respondeu por $19,1 \%$ dos atendimentos, acentuando a preocupação com as competências em saúde disponibilizadas no modelo. Considerando a Lei 7498/1986(23), a assistência direta a pacientes no APH só poderia ser realizada pelo Enfermeiro e/ou pelo Técnico de Enfermagem.

Já há em atividade no Brasil um padrão de prática para os enfermeiros no APH. Registros institucionais ${ }^{(24-25}$ e a vivência dos autores sobre a realidade nacional, destacam múltiplas experiências em serviços existentes nos estados de São Paulo(24), Rio de Janeiro, Bahia e Ceará(25), onde o enfermeiro atua como 3 으 profissional do SBV ou, na ausência do médico no SAV, formando uma equipe com o técnico de enfermagem ${ }^{(5,24,25)}$.

No SAMU 192 da cidade de São Paulo, o Enfermeiro atua desde 2004 com protocolo institucional(24) que registra medicações e procedimentos a serem utilizados com apoio do médico regulador e com uso da telemedicina. No geral, tais práticas se relacionam às necessidades de estabilização dos agravos tempo-dependentes e de agudização de doenças crônical incluindo medicações por via endovenosa ou intraóssea, manejo de vias aéreas com acesso supraglótico e utilização de escalas de avaliação. A indisponibilidade desses procedimentos em tempo oportuno, potencialmente concorreriam para a persistência e agravamento de alguns quadros, ocorrência de sequelas e maiores custos de internação para o sistema.

É preciso analisar as experiências nacionais e internacionais. Estudos sobre a complexidade dos atendimentos e a atuação da Enfermagem e dos Médicos no APH, segundo padrão, interação e resultados, são importantes para direcionar o desenvolvimento do modelo brasileiro.

A maior participação do enfermeiro na assistência, pode ser uma grande ferramenta de acesso oportuno, expandindo quali-quantitativamente o modelo de atenção nos grandes centros e nas áreas rurais e remotas, e também ampliando a satisfação das expectativas e ne- 
cessidades do usuário, resultando em impacto para o cidadão e para o sistema de saúde(5)

\section{Limitações do estudo}

Os estudos sobre resultados da EPA são mais frequentes na APS onde esse papel da Enfermagem já está consolidado. Análises sobre os resultados da participação do enfermeiro no APH ainda são limitados à algumas experiências no mundo, porém vem crescendo e se constituem em importante perspectiva de pesquisa. A revisão narrativa não se utiliza de estratégias sofisticadas e exaustivas de busca, e assim, a seleção dos estudos e a interpretação das informações está sujeita a maior subjetividade.

\section{Contribuições do estudo para a prática}

A expansão do papel do Enfermeiro é uma estratégia de ampliação do acesso à saúde. A fundamentação teórica, bem como a análise das experiências e dos contextos em que essa expansão pode ser útil, auxiliam na identificação de novas perspectivas de atuação profissional, ao mesmo tempo que apresentam modelos que podem servir de referência no enfrentamento dos problemas do sistema brasileiro de atenção à saúde.

\section{CONCLUSÃO}

Experiências internacionais demonstram que a EPA é uma estratégia de valor na busca de acesso à saúde. Frente ao cenário desafiador das urgências no Brasil, a incorporação de Enfermeiros treinados e habilitados no $\mathrm{APH}$, têm potencial para qualificar o modelo e levar segurança assistencial às áreas que ainda precisam ser cobertas, bem como, às áreas que vivenciam a indisponibilidade de médicos e/ou a exclusiva presença de SBV, garantindo acesso oportuno. A presença do enfermeiro amplia a capacidade de avaliação e possibilita o uso de medicações e procedimentos sob protocolos e regulação médica com o auxílio de telemedicina, trazendo benefícios ao paciente em situação de urgência e ao sistema de saúde, com um cuidado mais seguro, encaminhamentos mais adequados e possivelmente melhores resultados de saúde. A presença do enfermeiro no $\mathrm{APH}$ já é um fato relevante no mundo e em nosso país.

Contribuição dos autores: Concepção do artigo: MAAM, SDM, EFS; Análise e interpretação dos dados, Redação do artigo e Redação final : MAAM; Revisão Crítica: SDM, EFS, LPBB, MASF, LS, GCH, RB.

\section{REFERÊNCIAS}

1. Delamaire M, Lafortune, G. Nurses in Advanced Roles: a description and evaluation in 12 developed countries. Organisation for Economic Co-operation and Development (OECD). Health Working Papers. [Internet] 2010 [cited 2017 feb 13]; 54. Available from: http://www.oecd-ilibrary.org/docserver/ download $/ 5 \mathrm{kmbrcfms} 5 \mathrm{~g} 7$-enpdf?expires=1488735304Eid=idEacc name=guest8checksum=69DAF99FCAA32B3EF3D77153888E6F14

2. Sherr B, Wong FKY. The Development of Advanced Nursing Practice Globally. Journal of Nursing Scholarship. [Internet] 2008 [cited 2017 feb 13]; 40(3):204-11. Available from: http:// onlinelibrary.wiley.com/doi/10.1111/j.1547-5069.2008.00242.x/ epdf.

3. Bryant-Lukosius D, Valaitis R, Martin-Misener R, Donald F, Morán Peña L, Brousseau L. Advanced Practice Nursing: A Strategy for Achieving Universal Health Coverage and Universal Access to Health. Rev. Latino-Am. Enfermagem. [Internet] 2017 [cited 2018 Feb 13]: 25, e2826. Avaiable from: http://www.scielo.br/pdf/rlae/ v25/0104-1169-rlae-25-02826.pdf.

4. Cassiani SHDB, Zugi KE. Promovendo o papel da Prática Avançada de Enfermagem na América Latina. Rev Bras Enferm [Internet] 2014 [Cited 2017 Feb 13]; 67(5):675-6. Available from: http://www. scielo.br/pdf/reben/v67n5/pt_0034-7167-reben-67-05-0677.pdf 5. Malvestio MAA, Arci MS, Souza EF, Paula CE, Martuchi SD, Matos EFS, Veiga AG. Enfermagem em Práticas Avançadas: perspectivas para o atendimento pré-hospitalar. Enfermagem em Revista. 2017 [cited 2018 Jul 12]; 18:18-9. Disponivel em: https://portal. coren-sp.gov.br/wp-content/uploads/2015/11/revista_coren_ sp_junho_2017.pdf.

6. Pan American Health Organization (PAHO). Expanding the Roles of Nurses in Primary Health Care [Internet]. 2018 [cited $2018 \mathrm{Jul}$ 12]. Avaiable from: http://iris.paho.org/xmlui/ bitstream/handle/123456789/34958/9789275120033_eng. pdf?sequence $=6$ \&isAllowed $=y$

7. Toso, BRGO. Práticas Avançadas de Enfermagem em atenção primária: estratégias para implementação no Brasil. Enferm Foco [Internet]. 2016 [cited 2019 mar 02]: 7 (3/4): 36-40. Avaiable from: http://revista.cofen.gov.br/index.php/enfermagem/article/ view/913/343

8. Souza MFM, Malta DC, França EB, Barreto ML. Transição da saúde e da doença no Brasil e nas Unidades Federadas durante os 30 anos do Sistema Único de Saúde. Ciênc. saúde coletiva [Internet]. 2018 [cited 2019 mar 02]; 23(6):1737-1750. Avaiable from: http:// 
www.scielo.br/pdf/csc/v23n6/1413-8123-csc-23-06-1737.pdf

9. Ministério da Saúde (BR). Portaria nol600 GM/MS de 07 de julho de 2011. Reformula a Politica Nacional de Atenção às Urgências e institui a Rede de Atenção às Urgências no Sistema Único de Saúde (SUS). Diário Oficial da União. 07 jul. 2011. Available from: http://www.saude.pr.gov.br/arquivos/File/Portaria_n_1600_ de_07_07_11_Politica_Nac_Urg_Emerg.pdf. Cited 2018 dec 12

10. Mendes, EV. As redes de atenção à saúde. Brasilia: Organização pan-americana de Saúde, 2011.549p. [Cited 2018 dec 12]. Disponivel em: http://bvsms.saude.gov.br/bvs/publicacoes/ redes_de_atencao_saude.pdf

11. Newhouse RP, Stanik-Hutt J, White KM, Johantgen M, Bass EB, Zangaro G, et al. Advanced practice nurse outcomes 1990-2008: a systematic review. Nursing Economics [Internet]. 2011 [cited 2018 mar 02]; 29(5):1-22. Avaiable from: https://www.micnp.org/assets/ Website/StandardsofPracticePage/APN\%20outcomes\%20-\%20 A\%20Systematic\%20Review.pdf

12. Instituto Nacional de Emergência Médica (INEM). Relatório Anual Integração VMER e SIV 2017. 2017 [cited 2019 Feb 20]. Avaiable from:: https://www.inem.pt/wp-content/ uploads/2018/05/Relat\%C3\%B3rio-Integra\%C3\%A7\%C3\%B5esVMER-e-SIV-2017_vers\%C3\%A3o-corrigida-16-05-2018.pdf

13. Skog S, Linda W, Lindström V. Development of the Prehospital Emergency Care, The Registered Nurses' Role in the Ambulance Service - A Swedish perspective. Emergency Med [Internet]. 2015 [cited 2018 nov 18]; 6:294. Avaiable from: https://pdfs.semanticscholar. org/376d/693ca25302840e46fla3lf202d40ff37f4ee.pdf? _ $g a=2.159223098 .1844146819 .1581539126-1844378312.1562786353$

14. Van Schuppen H, Bierens J. Understanding the prehospital physician controversy. Step 1: comparing competencies of ambulance nurses and prehospital physicians. Eur $\mathrm{J}$ Emerg Med [Internet]. 2011[cited 2018 nov 18]; 18(6):322-7. Avaiable from: $\quad$ https://www.researchgate.net/publication/50989613_ Understanding_the_prehospital_physician_controversy_ Step_1_Comparing_competencies_of_ambulance_nurses_and_ prehospital_physicians

15. Eckstein M, Ito T, Guggenhein A, Sanko S. Nurse Practitioner Response Unit Launched in Los Angeles. Journal of Emergency Medical Services [Internet]. 2017 [cited 2018 nov 18], 2(42). Avaiable from: https://www.jems.com/articles/print/volume-42/ issue-2/features/nurse-practitioner-response-unit-launched-inlos-angeles.html

16. Ministério da Saúde (BR). Portaria no 2048 do, de 05 de novembro de 2002. Dispõe sobre o regulamento técnico dos sistemas estaduais de urgência e emergência. Brasilia, DF, 2002.
Available from: http://bvsms.saude.gov.br/bvs/saudelegis/ gm/2002/prt2048_05_11_2002.html. Cited 2016 dec 12

17. Ministério da Saúde (BR). Portaria de Consolidação no 3 GM/ MS de 03 de outubro de 2017. Consolida as normas sobre as redes do Sistema Único de Saúde. Brasilia, DF, 2017. Avaiable from: http://bvsms.saude.gov.br/bvs/saudelegis/gm/2017/ MatrizesConsolidacao/Matriz-3-Redes.html. Cited: 2018 mar 20.

18. Ministério da Saúde (BR) [Internet]. Dados de 2018: População passa a contar com 476 novas ambulâncias do SAMU 192 [Cited 10 dez 2018]. Avaiable from : http://www.saude.gov.br/noticias/ agencia-saude/42892-populacao-passa-a-contar-com-476novas-ambulancias-do-samu-192

19. Instituto Brasileiro de Geografia e Estatística. Infraestrutura Nacional de Dados Espaciais (INDE) [database]. Cited 2018 dec 10. Disponivel em: https://inde.gov.br/

20. Ministério da Saúde(BR). Sala de Apoio à gestão estratégica (SAGE) [database]. Cited 2018 nov 12. Avaiable from: http://sage. saude.gov.br/\#

30. O'Dwyer G et al. Implantação do Serviço de Atendimento Móvel de Urgência no Brasil: Estratégias de ação e dimensões estruturais. Cad. Saúde Pública. 2017 [cited 2018 Mar 13]; 33(7):e00043716. Avaiable from:http://www.scielo.br/pdf/csp/ v33n7/1678-4464-csp-33-07-e00043716.pdf.

31. Ministério da Saúde(BR). Tabnet Datasus [database]. Cited 2019 apr 15. Avaiable from: http://tabnet.datasus.gov.br/cgi/ tabcgi.exe?sia/cnv/qauf.def.

32. Presidência da República (BR). Lei no 7498 de 25 de junho de 1986. Dispõe sobre a regulamentação do exercício da Enfermagem e dá outras providências. Brasilia, DF 1986. Avaiable from: http:// www.cofen.gov.br/lei-n-749886-de-25-de-junho-de-1986_4161. html. Cited 2018 mar 20

33. Secretaria Municipal da Saúde de São Paulo (BR). SAMU 192 Protocolos deAtendimento Pré-hospitalar Suporte Intermediário de Vida [Internet]. 2014 [cited 2018 mar 13]. Avaiable from: https://www.prefeitura.sp.gov.br/cidade/secretarias/upload/ saude/protocolodeatendimentoprehospitalar.pdf Acessado em $13 / 03 / 2018$

34. Secretaria Municipal de Saúde de Fortaleza (BR). SAMU 192 Regional Fortaleza. Protocolos de Suporte Básico de Vida SAMU-For [Internet].2016 [cited 2018 mar 20]. Avaiable from: http://www.samu.fortaleza.ce.gov.br/index.php/repositorioinstitucional/send/2-normas-de-conduta-tecnica-egestora/85-protocolos-de-suporte-basico-de-vida-samufor

RECEBIDO EM: 01/09/2019. - ACEITO EM:16/12/2019 